\title{
Clinical study of recurrent mild encephalitis/ encephalopathy with a reversible splenial lesion in two cases
}

\author{
Jiao Xue ${ }^{1}$, Chongfeng Duan ${ }^{2}$, Guizhi Xu ${ }^{3}$, Ying Zhang ${ }^{1}$, Zhenfeng Song ${ }^{1}$, Zhi Yi ${ }^{1}$, Chengqing Yang ${ }^{1}$, Fei Li ${ }^{1}$, \\ Kaixuan Liu ${ }^{1}$, Hongshan Zhao ${ }^{4}$ and Xuejun Liur ${ }^{2 *}$
}

\begin{abstract}
Background: Mild encephalitis/encephalopathy with a reversible splenial lesion (MERS) has been reported worldwidely. However, the data about recurrent cases is limited. We aimed to analyze the clinical and radiographic features of recurrent MERS, and its possible mechanisms.

Case presentation: Two patients with clinically recurrent MERS were reported here, exhibiting neurological symptoms such as limbs weakness and numbness, stand/walk unsteadily, slurred speech and irritability, and typical lesions in the corpus callosum and white matter. One of them experienced another four episodes with a similar clinical course and magnetic resonance imaging findings over a period of 10 years. The Na levels in the present two patients were normal.

Discussion and conclusion: Combined with the patients reported previously, recurrence could be seen in both MERS type 1 and type 2 patients, from two to multiple times, with the latter possibly more common. It suggested that some genetic factors might be involved in MERS, especially for MERS type 2 or familial MERS.
\end{abstract}

Keywords: Child, Recurrent, MERS

\section{Background}

Mild encephalitis/encephalopathy with a reversible splenial lesion (MERS) is a clinico-radiological syndrome first described by Tada et al. [1] at 2004, characterized by transient lesions in the corpus callosum on magnetic resonance imaging (MRI) and mild encephalopathy following prodromal symptoms such as fever, cough, vomiting and/or diarrhea, which usually recover within a month [2]. It was further proposed to be classified into MERS type 1, with an isolated lesion in the splenium of the corpus callosum, and MERS type 2, with an extensive white

\footnotetext{
*Correspondence: bncz@sina.com

${ }^{2}$ Department of Radiology, the Affiliated Hospital of Qingdao University,

Qingdao, No. 1677 Wutaishan Road, Qingdao, Shandong 266000, PR

China

Full list of author information is available at the end of the article
}

matter and/or entire callosal lesions [3]. The pathogenesis of MERS is still not fully understood. Many studies of MERS have been reported in recent years, involving clinical and imaging features, as well as triggering factors, etc. [2, 4-6]. However, research on recurrent MERS is extremely rare. Here, we reported the clinico-radiological features of 2 cases with recurrent MERS, and discussed the possible pathogenic mechanisms.

\section{Case presentation \\ Case 1}

In April 2016, a previously healthy 11-year-old boy was admitted to our hospital due to intermittent right upper limb weakness, numbness in the right corner of the mouth, irritability and then anepia and stand unsteadily sometimes, lasting for several hours. There was no 
family history or past history of neurological disorders, including epilepsy and developmental retardation. The results of neurological examination were unremarkable. Blood examinations revealed a normal white blood cell count $\left(5.80 \times 10^{\wedge} 9 / \mathrm{L}\right)$ and C-reactive protein level $(0.90 \mathrm{mg} / \mathrm{L})$, and normal levels of ammonia, glucose and $\mathrm{Na}(138 \mathrm{mmol} / \mathrm{l})$. Anti-nuclear antibody and antineutrophils cytoplasmic antibody were normal also. Cerebrospinal fluid (CSF) examinations showed normal cell counts, and protein and glucose levels. Cranial MRI on day 2 revealed lesions in the entire corpus callosum and diffuse white matter with moderate hyper intensity on T2-weighted images, and marked hyperintensity on diffusion-weighted images (DWI) (Fig. 1A-F). A diagnosis of MERS type 2 was considered according to the clinical and radiological features. He was treated with intravenous methylprednisone and antiviral therapy; his clinical manifestations gradually improved, and he had completely recovered by day 5 . Follow-up MRI on day 12 showed resolution of the lesions on T2 and DWI (Fig. 1G-J).

In August 2018, he was admitted to our hospital again because of right limb weakness and numbness with right face numbness and a feeble voice, and then left limb weakness and numbness. The results of neurological examination were unremarkable. Blood examinations showed no special abnormalities. Serum $\mathrm{Na}(142 \mathrm{mmol} / \mathrm{l})$ was normal. Electroencephalography (EEG) was also normal with no epileptic discharges or high voltage slow waves. Cranial MRI showed similar lesions in the entire corpus callosum and diffuse white matter as before
(Fig. 2A-D). He was considered as recurrent MERS type 2 and treated with antiviral drug and mecobalamine to nourish the nerve. His clinical manifestations completely recovered by day 3 . Then a follow-up MRI on day 18 showed resolution of the lesions (Fig. 2E-H).

\section{Case 2}

In August 2010, a previously healthy 5-year-old boy was admitted to our hospital for slurred speech, limbs weakness, walk unsteadily with tumble sometimes. He had a prodromal infection of cough, fever, vomit and headache 2 weeks before this event. There was no family history or past history of neurological disorders. He had received antibiotics (ampicillin, cefuroxime and azithromycin) and Ibuprofen before admission. On admission, the muscle strength and tone of the limbs were normal. The results of neurological examination showed mild positive of Kernig sign, Babinski sign and Chaddock sign. Blood examinations revealed a mild elevated white blood cell count $\left(11.08 \times 10^{\wedge} 9 / \mathrm{L}\right)$ and normal C-reactive protein $(0.49 \mathrm{mg} / \mathrm{L})$, glucose and $\mathrm{Na}(139 \mathrm{mmol} / \mathrm{l})$ level. Cranial MRI revealed lesions in the splenium of the corpus callosum and diffuse white matter including semi-ovoid center and periventricular with moderate hyper intensity on T2-weighted images, and marked hyperintensity on DWI (Figures unavailable). Cervical, thoracic and lumbar MRI were normal. MERS has not widely recognized at that time. He was diagnosed as encephalitis post-infected and was treated with intravenous methylprednisone, anti-infective therapy (ganciclovir and cefepime) and mannitol. His

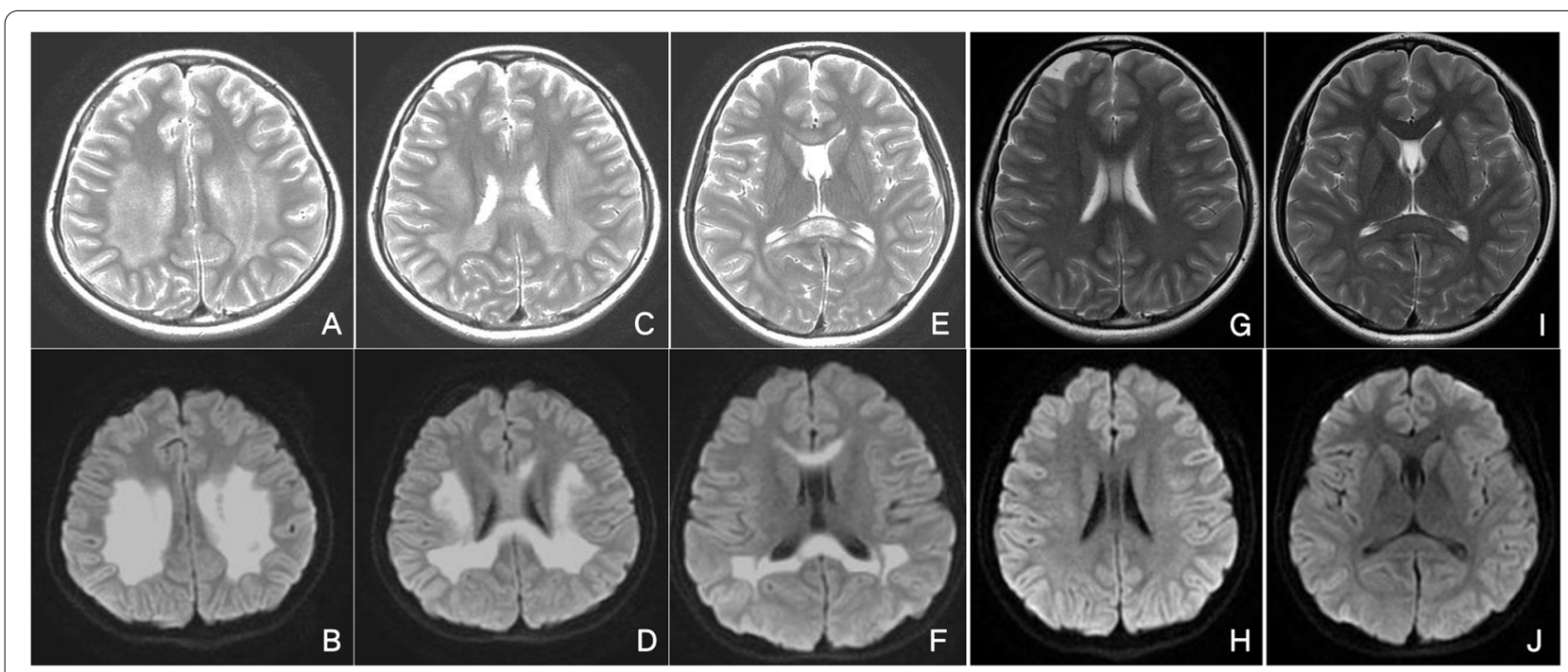

Fig. 1 Cranial MRI of case 1 in 2016. Cranial MRI on day 2 showed lesions in the entire corpus callosum and diffuse white matter with moderate hyper intensity on T2-weighted images $(\mathbf{A}, \mathbf{C}, \mathbf{E})$ and marked hyperintensity on DWI (B,D,F). Follow-up MRI on day 12 showed resolution of the lesions on T2 $(\mathbf{G}, \mathbf{I})$ and DWI $(\mathbf{H}, \mathbf{J})$ 


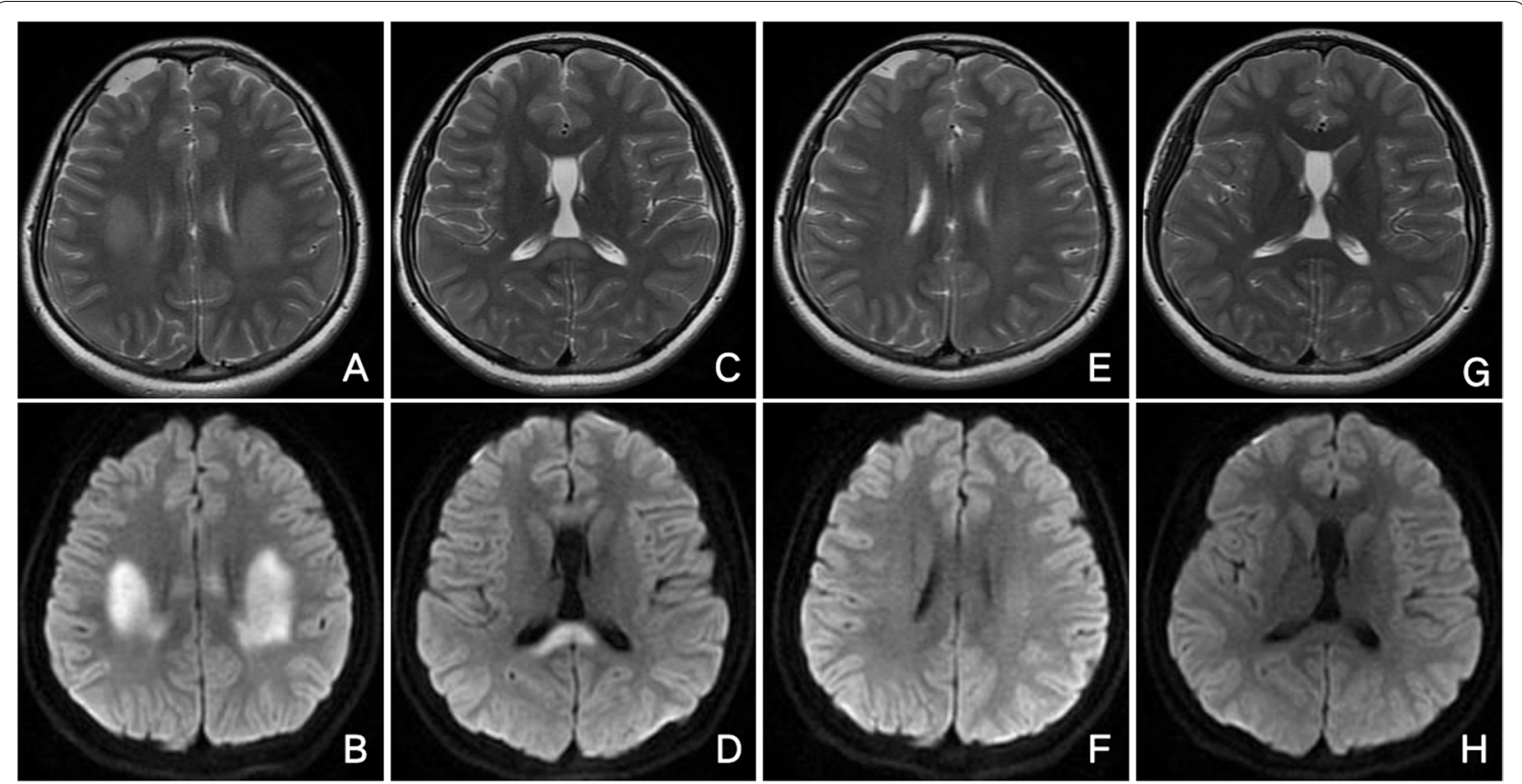

Fig. 2 Cranial MRI of case 1 in 2018. Initial cranial MRI showed lesions in the entire corpus callosum and diffuse white matter on T2-weighted images $(\mathbf{A}, \mathbf{C})$ and DWI (B,D). Follow-up MRI on day 18 showed resolution of the lesions on T2 $(\mathbf{E}, \mathbf{G})$ and DWI $(\mathbf{F}, \mathbf{H})$

clinical manifestations gradually improved, and he had completely recovered by day 4 , with a negative neurological examination. Follow-up MRI on day 15 showed a resolution of the lesion in the splenium of the corpus callosum and diminished lesion in periventricular matter with moderate hyper intensity on T2-weighted images but normal on DWI (Figures unavailable).
And then he experienced four episodes with a similar clinical course and MR imaging findings over a period of 10 years (in 2012, 2015, 2017 and 2020 yeas, respectively) (Fig. 3A-F; Fig. 4A-F), and was considered as recurrent MERS type 2. The clinical manifestations disappeared within several days under the treatment of intravenous methylprednisone. And the cranial MRI

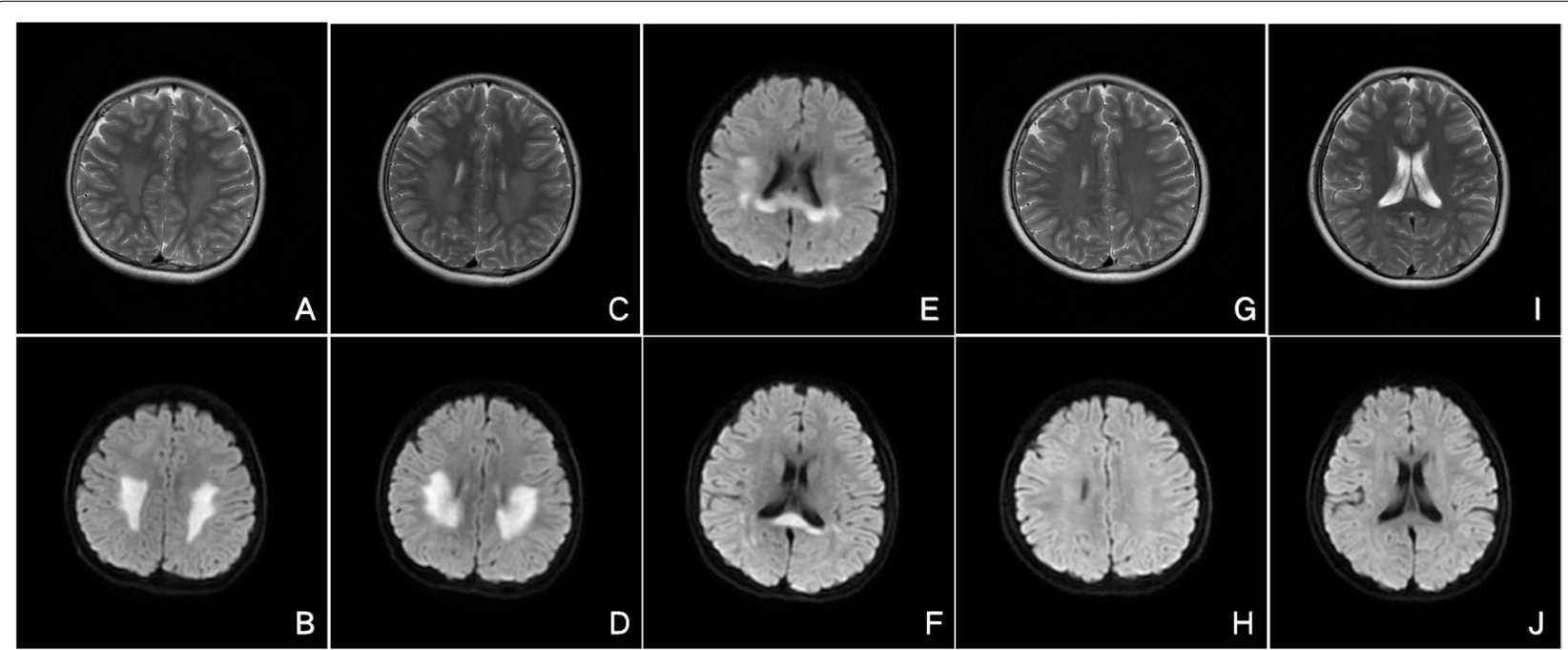

Fig. 3 Cranial MRI of case 2 in 2015. Initial cranial MRI showed lesions in the entire corpus callosum and diffuse white matter on T2-weighted images $(\mathbf{A}, \mathbf{C})$ and DWI $(\mathbf{B}, \mathbf{D}-\mathbf{F})$. Follow-up MRI showed resolution of the lesions on T2 $(\mathbf{G}, \mathbf{I})$ and DWI $(\mathbf{H}, \mathbf{J})$ after seven weeks 


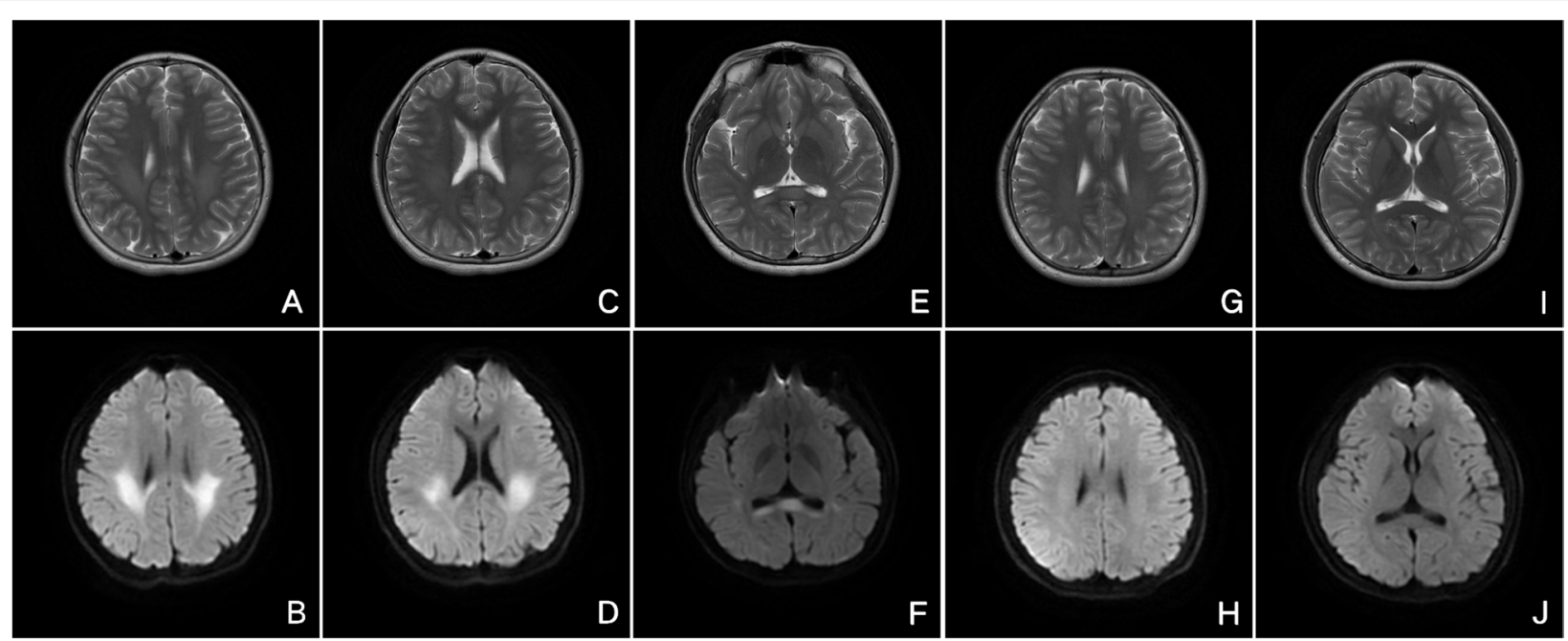

Fig. 4 Cranial MRI of case 2 in 2017. Initial cranial MRI showed lesions in the entire corpus callosum and diffuse white matter T2-weighted images $(\mathbf{A}, \mathbf{B}, \mathbf{E})$ and marked hyperintensity on DWI (B,D,F). Follow-up MRI showed resolution of the lesions on T2 $(\mathbf{G}, \mathbf{I})$ and DWI $(\mathbf{H}, \mathbf{J})$ after 3 months

after several weeks showed resolution of the lesions completely (Fig. 3G-J; Fig. 4G-J). He had normal psychomotor development without any neurological sequelae.

\section{Discussion and conclusion}

MERS is a subtype of acute encephalopathy state with characteristic diagnostic MRI findings suggesting myelin vacuolization $[1,7]$. Although a great number of cases has been reported worldwidely, the data about recurrent MERS is limited to a really few case report. In this report, we described two cases with recurrent MERS type 2, and one of them had multiple relapses. Although the cranial MRI of one patient (case 2) was not normal completely at discharge, it was reasonable to speculate that the lesion would disappear with time given the significant reduction in lesion scope and signal intensity, as well as the followed similar episodes.

After browsing in Wanfang, CNKI and PubMed database, as well as searching the references for related published articles, we found only several cases with recurrent MERS in literatures. Dong et al. [8] reported a girl who experienced repeated episodes of convulsions at 19 and 21 months of age, following cough and vomit respectively. The serum Na was normal. After anti-infective and glucocorticoid treatment, clinical symptoms disappeared within a few days. Both cranial MRI revealed a lesion in the splenium of the corpus callosum at first and disappeared on day 7 and day 8 , respectively. And a diagnosis of MERS type 1 was confirmed. Duan et al. [9] reported a boy with recurrent MERS type 2, which was included in our study here (case 1). Moreover, Imamura et al. [10] mentioned that a 5-year-old girl who experienced MERS three times and Zhang et al. [11] described a child who experienced MERS two times. However, neither specific clinical nor imaging description of these two patients was offered. Besides, Shigeno et al. [12] reported a rare case of MERS associated with transient ischemic attact (TIA)like symptoms. He presented with transient left hemiparesis and dysarthria at 9 years old and was diagnosed with MERS type 2 due to the typical clinical course and MRI findings. Then he had experienced a similar symptom at 13 years old and a diagnosis of recurrence of MERS type 2 was considered. And then he experienced eight TIA-like episodes with a similar clinical course and MRI findings over a period of 6 years. It was worth noting that one of our patients (case 1) was mainly characterized by unilateral limb weakness and numbness, which was similar to the TIA-like symptoms described by Shigeno et al. [12]. If so, the possibility of multiple recurrences cannot be ruled out and further follow-up is needed. Combined with the patients reported previously and our two patients, it suggests that both MERS type 1 and MERS type 2 are likely to relapse, one or even more times. It seems that patients with type 2 may be more likely to relapse, but in view of the small number of cases, no firm conclusions can be drawn yet.

The exact pathomechanism of MERS remains unclear. PPossible mechanisms include intramyelinic edema, a transient inflammatory infiltrate, and interstitial edema in tightly packed fibers $[1,2]$. Laboratory evaluation revealed that hyponatremia is common in patients with MERS [13]. Hypotonic hyponatremia causes water to enter the brain, resulting in cerebral edema. The $\mathrm{Na}$ levels in the present two patients, as well as in the 
patient reported by Dong et al. [8], however, were normal, which suggested that the pathophysiology of the recurrent MERS might be different from that of sporadic MERS.

Kurahashi et al. [14] reported a family with recurrent encephalopathy spanning over three generations, which presented with extensive but reversible cerebral myelin vacuolization and neurological symptoms similar MERS. And a heterozygous c.1208A > G (p.Gln403Arg) in the myelin regulatory factor (MYRF) gene was identified $[10,14]$. As a transcriptional regulator, MYRF is significant for oligodendrocyte differentiation and myelin maintenance [15]. Therefore, functional deficiency of MYRF may be causally related to extensive myelin vacuolation [16]. Although Kurahashi et al. [14] suggested that MYRF variant cases and sporadic MERS cases might be different conditions. They also mentioned that the central nervous system (CNS) symptoms of MYRF variant cases were actually indistinguishable from MERS, and MRI features involving widespread white matter and the entire corpus callosum were also very similar to the MERS type 2 , which suggested that MYRF variant cases might be a more extensive form of MERS. Meanwhile, the MYRF variant was also identified in another family that they partially described as familial MERS-like features (MERS type 2?) [10]. Therefore, it is reasonable to believe that some genetic factors might be involved in MERS, especially for MERS type 2 or familial MERS.

In conclusion, recurrence could be seen in both MERS type 1 and type 2 patients, from two to multiple times, with the latter possibly more common. Recurrent and/or familial cases with a clinical diagnosis of MERS suggest the presence of genetic factors. Further clinical, radiological and genetic studies are needed for a definite conclusion.

\footnotetext{
Abbreviations

MERS: Mild encephalitis/encephalopathy with a reversible splenial lesion; MRI: Magnetic resonance imaging; CSF: Cerebrospinal fluid; DWl: Diffusionweighted images; EEG: Electroencephalography; TIA: Transient ischemic attact; MYRF: Myelin regulatory factor.
}

\section{Acknowledgments}

We thank the patients and their families for participating.

\section{Authors' contributions}

Conception and design of the study: JX, ZS, XL; Acquisition and analysis of data: CD, GX, YZ, ZS, ZY, CY, FL, KL, HZ; Drafting a significant portion of the manuscript or figures: JX. All authors have read and approved the manuscript.

\section{Funding}

This work including data collection, analysis and follow-up was supported by the Taishan Scholars Program of Shandong Province (NO.tsqn201909191), Youth Fund of the Affiliated Hospital of Qingdao University (NO.

QDFYQN2020014) and Youth Fund of Shandong Natural Science Foundation (NO.ZR2021QH042).

\section{Availability of data and materials}

Datasets used and/or analysed during the current study are available from the corresponding author on reasonable request.

\section{Declarations}

Ethics approval and consent to participate

The study was approved by the Ethical Committee of Affiliated Hospital of Qingdao University. Written informed consent was obtained from the parents/ guardians of the patient for publication of this case report and any accompanying images. A copy of the written consent is available for review by the Editor of this journal.

\section{Consent for publication}

Both of the parents/guardians gave their written consent for their child's personal or clinical details along with any identifying images to be published in this study.

\section{Competing interests}

The authors have no potential conflicts of interest to disclose.

\section{Author details}

${ }^{1}$ Department of Pediatric Neurology, the Affiliated Hospital of Qingdao University, Qingdao 266000, Shandong, China. ${ }^{2}$ Department of Radiology, the Affiliated Hospital of Qingdao University, Qingdao, No. 1677 Wutaishan Road, Qingdao, Shandong 266000, PR China. ${ }^{3}$ Department of Radiology, Zhucheng People Hospital, Weifang 262200, Shandong, China. ${ }^{4}$ Department of Anesthesiology, the Affiliated Hospital of Qingdao University, Qingdao 266000, Shandong, China.

Received: 10 June 2021 Accepted: 23 December 2021

Published online: 03 January 2022

\section{References}

1. Tada H, Takanashi J, Barkovich AJ, Oba H, Maeda M, Tsukahara H, et al. Clinically mild encephalitis/encephalopathy with a reversible splenial lesion. Neurology. 2004;63:1854-8.

2. Takanashi J. Two newly proposed infectious encephalitis/ encephalopathy syndromes. Brain and Development. 2009;31(7):521-8.

3. Takanashi J, Imamura A, Hayakawa F, Terada H. Differences in the time course of splenial and white matter lesions in clinically mild encephalitis/ encephalopathy with a reversible splenial lesion (MERS). J Neurol Sci. 2010;292(1-2):24-7.

4. Hoshino A, Saitoh M, Oka A, Okumura A, Kubota M, Saito Y, et al. Epidemiology of acute encephalopathy in Japan, with emphasis on the association of viruses and syndromes. Brain and Development. 2012;34(5):337-43.

5. Yıldız AE, Maraş Genç H, Gürkaş E, Akmaz Ünlü H, Öncel İH, Güven A. Mild encephalitis/encephalopathy with a reversible splenial lesion in children. Diagn Interv Radiol. 2018;24(2):108-12.

6. Pan JJ, Zhao YY, Lu C, Hu YH, Yang Y. Mild encephalitis/encephalopathy with a reversible splenial lesion: five cases and a literature review. Neurol Sci. 2015;36(11):2043-51.

7. Takanashi J, Barkovich AJ, Yamaguchi K, Kohno Y. Influenza-associated encephalitis/encephalopathy with a reversible lesion in the splenium of the corpus callosum: a case report and literature review. AJNR Am J Neuroradiol. 2004;25(5):798-802.

8. Dong Y, Guo A, Liu L, Wang Y, Chen N, Li J. Clinical study of mild encephalitis/encephalopathy with a reversible lesion in the splenium of the corpus callosum in children. Clin Pediatr Emerg Med. 2017;24(9):709-12.

9. Duan C, Li N, Niu L, Zhao J, Liu F, Zhang S, et al. Recurrent type II mild encephalitis/encephalopathy with reversible splenial lesion: a case report. Chin J Neurol. 2020;53(4):305-8.

10. Imamura T, Takanashi J, Yasugi J, Terada H, Nishimura A. Sisters with clinically mild encephalopathy with a reversible splenial lesion (MERS)-like features; familial MERS? J Neurol Sci. 2010;290(1-2):153-6.

11. Zhang C, Li G, Zhang X, Li C. Clinical manifestations and imaging features of slight encephalitis children complicated with reversible lesions of 
Corpus callosum Splenium. Pract I Cardiac Cerebral Pneumal Vasc Dis. 2017;25(9):82-5.

12. Shigeno A, Hiu T, Iwanaga $H$, Yasu T, Honda R, Nakaoka K, et al. A pediatric case of clinically mild encephalitis/encephalopathy with a reversible Splenial lesion (MERS) with recurrent TIA-like symptoms over a prolonged period. No Shinkei Geka. 2020;48(2):131-40.

13. Takanashi J, Tada H, Maeda M, Suzuki M, Terada H, Barkovich AJ. Encephalopathy with a reversible splenial lesion is associated with hyponatremia. Brain and Development. 2009;31(3):217-20.

14. Kurahashi H, Azuma Y, Masuda A, Okuno T, Nakahara E, Imamura T, et al. MYRF is associated with encephalopathy with reversible myelin vacuolization. Ann Neurol. 2018;83(1):98-106.

15. Emery B, Agalliu D, Cahoy JD, Watkins TA, Dugas JC, Mulinyawe SB, et al. Myelin gene regulatory factor is a critical transcriptional regulator required for CNS myelination. Cell. 2009;138(1):172-85.

16. Koenning M, Jackson S, Hay CM, Faux C, Kilpatrick TJ, Willingham M, et al. Myelin gene regulatory factor is required for maintenance of myelin and mature oligodendrocyte identity in the adult CNS. J Neurosci. 2012;32(36):12528-42.

\section{Publisher's Note}

Springer Nature remains neutral with regard to jurisdictional claims in published maps and institutional affiliations.

- fast, convenient online submission

- thorough peer review by experienced researchers in your field

- rapid publication on acceptance

- support for research data, including large and complex data types

- gold Open Access which fosters wider collaboration and increased citations

- maximum visibility for your research: over $100 \mathrm{M}$ website views per year

At BMC, research is always in progress.

Learn more biomedcentral.com/submissions 